\title{
CONTRIBUIÇÕES DO PRONTUÁRIO ELETRÔNICO PARA PACIENTES, PROFISSIONAIS E INSTITUIÇÕES DE SAÚDE: UMA REVISÃO INTEGRATIVA
}

\author{
CONTRIBUTIONS FROM ELECTRONIC MEDICAL RECORDS FOR \\ PATIENTS, HEALTH PROFESSIONALS AND INSTITUTIONS: AN \\ INTEGRATIVE REVIEW
}

Michelinne Oliveira Machado Dutra ${ }^{1}$ Juliana Jamaica Sousa da Silva ${ }^{2}$ Michelly Guedes de Oliveira Araújo ${ }^{3}$

\begin{abstract}
RESUMO: Objetivo: analisar a produção científica acerca das contribuições do prontuário eletrônico para pacientes, profissionais e instituições de saúde. Método: revisão integrativa da literatura, realizada no mês de fevereiro e março de 2020, nas bases de dados PubMed, LILACS, SciELO e BVS. Sendo incluídos artigos escritos em inglês, espanhol e português. Os artigos selecionados foram gerenciados no programa JabRef Reference Manager 2.5. Resultados: Na busca inicial foram identificados 91 artigos, sendo incluídos 07 no estudo. Todos os manuscritos pertenciam à área de saúde e apresentavam contribuições para pacientes, profissionais e instituições de saúde. Conclusão: a análise dos artigos mostra-se de grande relevância, uma vez que evidencia que a utilização do prontuário eletrônico proporciona contribuições éticas, legais, aspectos de segurança, estímulo ao trabalho em equipe, aumento da qualidade e produtividade do serviço, integração de informações, atualização de dados, subsídio à pesquisa e auditoria.
\end{abstract}

Descritores: Prontuário Eletrônico. Informática em Saúde. Prestação de Cuidados de Saúde.

ABSTRACT: Objective: To analyze the scientific production about the contributions of electronic medical records for patients, health professionals and institutions. Method: integrative review of literature, held in the month of February and March of 2020, the databases PubMed, LILACS, SciELO and VHL. Being included articles written in English, Spanish and Portuguese. The articles selected were managed in

\footnotetext{
${ }^{1}$ Enfermeira. Doutora em Enfermagem. Docente do curso de Medicina da UNIFACISA.

2 Graduanda em Medicina pela UNIFACISA.

${ }^{3}$ Enfermeira. Doutoranda em Enfermagem pela UPE/UEPB.
} 
the program JabRef Reference Manager 2.5. Results: In the initial search were identified 91 articles, 07 were included in the study. All manuscripts belonged to the area of health and presented contributions to patients, health professionals and institutions. Conclusion: The analysis of the articles shows of great relevance, since it demonstrates that the use of electronic medical records provides contributions ethical, legal, security aspects, a stimulus to work in team, increasing productivity and quality of service, integration of information, updating of data allowance, to research and audit.

Keywords: Electronic Medical Records, Informatics in health, health care provision. 


\section{INTRODUÇÃO}

A expressão prontuário deriva-se do latim promptuarium, referente a um livro de anotações ordenadas em categorias, definidas em partes, objetivando simplificar o diagnóstico, proporcionar fiscalização da segurança e permitir auditória, ocasionando aumento da privacidade para profissionais e pacientes (LOURENÇÃO; JÚNIOR, 2016).

Nesse sentido, o Prontuário Eletrônico do Paciente (PEP) surge não apenas para assumir as funções típicas do prontuário em papel, mas também proporcionar melhoria na qualidade assistencial prestada em virtude de sua aplicabilidade e disposição de recursos, possibilidade de acesso síncrono e remoto de informações, segurança no armazenamento e transmissão de dados, layout flexível, integração com outros sistemas de informação, recuperação de dados para análise e auxílio na tomada de decisões pela equipe profissional (LOURENÇÃO; FERREIRA, 2016; MARTINS; LIMA, 2014).

Desse modo, o PEP é utilizado internacionalmente na assistência à saúde possibilitando o domínio eficiente e seguro de informações relativas a dados individuais e gerenciais do acompanhamento multiprofissional de clientes. É empregado ainda, como instrumento de administração, visando a contenção de despesas, direção, normalização de métodos assistenciais, subsidio à pesquisas e educação em saúde (PEREIRA et al., 2017).

Justifica-se o presente estudo em virtude do PEP ser um documento legítimo, responsável pelo armazenamento de dados pessoais de usuários dos serviços de saúde, tais como dados demográficos, histórico de saúde, procedimentos cirúrgicos, exames realizados, utilização de fármacos. Dados estes que favorecem a tomada de decisões por parte da equipe multiprofissional em saúde (MARTINS; LIMA, 2015).

Portanto, partindo do pressuposto de que o prontuário eletrônico proporciona contribuições para assistência à saúde, propõe-se a presente investigação com o 
objetivo de analisar a produção científica acerca das contribuições do prontuário eletrônico para pacientes, profissionais e instituições de saúde.

\section{MÉTODO}

Trata-se de uma revisão integrativa da literatura científica realizada entre os meses de fevereiro e março de 2020. A questão norteadora do estudo foi: Quais as contribuições do prontuário eletrônico para pacientes, profissionais e instituições de saúde? Para responder tal questionamento a busca dos manuscritos foi realizada via internet nas bases de dados PubMed (National Library of Medicine), LILACS (Literatura Latino-Americana e do Caribe em Ciências da Saúde), SciELO (Scientific Electronic Library Online) e BVS (Biblioteca Virtual em Saúde). Foram utilizados os seguintes descritores: "Prontuário Eletrônico"/ "Electronic Medical Records", "Informática em Saúde/ Medical Informatics", "Prestação de Cuidados de Saúde/ "Delivery of Health Care" extraídos dos Descritores em Ciências da Saúde (DeCS) e do Medical Subject Headings (MeSH) e o descritor não controlado "Contribuições". Foi utilizado o método de busca avançado, no qual cada descritor é considerado como um único termo e, estes, associados entre si por meio do operador booleano AND.

Os artigos foram selecionados de acordo com os seguintes critérios de inclusão: presença dos descritores escolhidos no título do trabalho ou inseridos no resumo; artigos na íntegra, disponíveis em texto completo; escritos nos idiomas português, espanhol ou inglês e publicados entre janeiro de 2013 a dezembro de 2019. Optou-se por este período de seleção em virtude da disponibilidade e concentração recente de publicações sobre a temática. Como critério de exclusão foram considerados os estudos de revisão e os artigos que se apresentaram duplicados.

Para coleta de dados foi utilizado um formulário adaptado de um instrumento já validado (GANONG, 1987). Foram coletadas informações referentes ao nome do autor, ano de publicação, área de conhecimento, base de dados, nome do periódico, 
título do artigo e tipo de estudo. Para catalogar os artigos será utilizado o Software JabRef Reference Manager versão 2.5.

Para avaliar a qualidade da evidência cientifica dos estudos selecionados foi utilizada a seguinte classificação: Nível 1: as evidências são provenientes de revisão sistemática ou metanálise de todos ensaios clínicos randomizados controlados relevantes; Nível 2: evidências derivadas de pelo menos um ensaio clínico randomizado controlado; Nível 3: evidências obtidas de ensaios clínicos bem delineados sem randomização; Nível 4: evidências provenientes de estudos de coorte e de caso controle bem delineados; Nível 5: evidências originárias de revisão sistemática de estudos descritivos e qualitativos; Nível 6: evidências derivadas de um único estudo descritivo ou qualitativo; Nível 7: evidências oriundas de opinião de autoridades e/ou relatório de comitês de especialistas (MELNYK; FINEOUTOVERHOLT, 2011).

As análises foram realizadas por meio da leitura, avaliação e agrupamento dos artigos (GANONG, 1987), alicerçadas no instrumento elaborado e seleção por meio dos critérios de inclusão e exclusão. Os achados foram apresentados na forma de quadros e na linguagem descritiva.

\section{RESULTADOS}

Foram encontrados 91 artigos nas bases de dados consultadas, sendo 61 na PubMed, 10 na LILACS, 09 na SciELO e 11 na BVS. Desses, 07 artigos preencheram os critérios de inclusão descritos acima e foram incluídos na amostra. Entre os 84 artigos descartados, 06 aparecem em mais de uma base de dados e os demais não atenderam ao primeiro requisito dos critérios de inclusão.

Neste estudo, foram incluídos 07 artigos que apresentaram evidências sobre as contribuições do prontuário eletrônico para pacientes, profissionais e instituições de saúde.

Conforme o fluxograma apresentado a seguir, observa-se que foram identificados 91 artigos, e selecionados 07 . Os manuscritos excluídos tiveram como 
justificativas: fuga da temática $(n=71)$, repetição em mais de uma base dados ( $n=$ 06) e serem revisão da literatura $(n=07)$ (Figura 1$)$.

Apresenta-se no Quadro 1, os periódicos que apresentaram publicação sobre as contribuições do prontuário eletrônico para pacientes, profissionais e instituições de saúde: "REUOL" (1), " Journal of Health Informatics" (1), " Saúde em Debate " (1), "Rev Bras Ter Intensiva" (1), " Cogitare Enfermagem" (1), "Appl Clin Inform" (1) e "Cadernos de Saúde Pública" (1). Quanto ao ano de publicação, foram observadas três publicações em 2013, duas em 2014, uma em 2016 e uma em 2018.

Em relação à área de conhecimento todos fazem parte da área de saúde com destaque para a Enfermagem com três artigos, Medicina com dois artigos e Psicologia, Informática e Odontologia com um artigo cada. (Quadro 1).

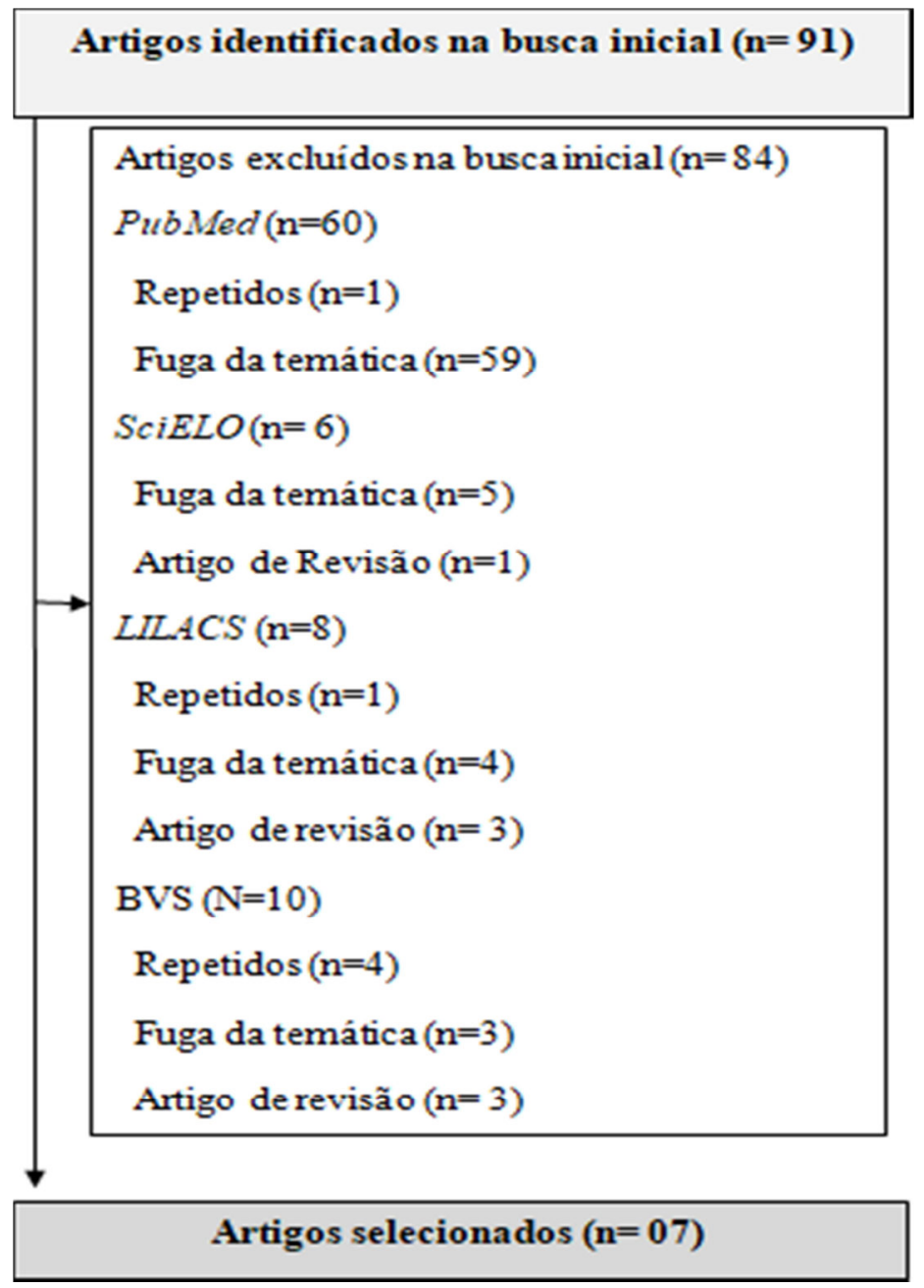

Figura 1 - Fluxograma da seleção dos artigos. 
Concernente ao cenário do estudo (Quadro 1), os artigos que compuseram a amostra são provenientes de serviços hospitalares (6), e Unidades Básicas de Saúde (2). Quanto ao local de origem dos estudos observa-se que sete foram realizados no Brasil e um no Teerã/Irã. Em relação à classificação da evidência seis artigos se enquadram no nível 6 e um artigo se enquadrou no nível 4.

Visualiza-se no Quadro 3 os resultados dos estudos com relação às contribuições advindas do uso do PEP para pacientes, profissionais e instituições de saúde, obtidos por meio da leitura e análise dos artigos, tomando por base os critérios explícitos e implícitos presentes nos mesmos.

Quadro 1 - Distribuição dos artigos segundo o nome dos autores, ano de publicação, área do conhecimento, cenário do estudo/local, nome do periódico e classificação de evidência.

\begin{tabular}{|c|c|c|c|c|}
\hline Autores/Ano & $\begin{array}{c}\text { Área do } \\
\text { conhecimento }\end{array}$ & $\begin{array}{l}\text { Cenário do } \\
\text { Estudo/Local }\end{array}$ & $\begin{array}{l}\text { Nome dos } \\
\text { periódicos }\end{array}$ & Evidência \\
\hline $\begin{array}{c}\text { ALVES; NOVAES } \\
2013\end{array}$ & Enfermagem & Recife- PE Hospital & REUOL & Nível 4 \\
\hline $\begin{array}{l}\text { NAMORATO, et al. } \\
2013\end{array}$ & Medicina & $\begin{array}{l}\text { Volta Redonda- } \\
\text { RJ/Hospita }\end{array}$ & $\begin{array}{l}\text { Journal of } \\
\text { Health } \\
\text { Informatics }\end{array}$ & Nível 6 \\
\hline $\begin{array}{c}\text { GONÇALVES, } 2013 \\
\text { et al. }\end{array}$ & $\begin{array}{c}\text { Medicina/ } \\
\text { Enfermagem }\end{array}$ & $\begin{array}{c}\text { Montes Claros- MG } \\
\text { Unidade Básica de } \\
\text { Saúde }\end{array}$ & Saúde em & Nível 6 \\
\hline FUMIS, et al. 2014 & Psicologia & $\begin{array}{c}\text { São Paulo-SP/ } \\
\text { Hospital }\end{array}$ & $\begin{array}{l}\text { Rev Bras Ter } \\
\text { Intensiva }\end{array}$ & Nível 6 \\
\hline $\begin{array}{c}\text { LAHM; CARVALHO } \\
2014\end{array}$ & Enfermagem & Sul do Brasil/Hospital & $\begin{array}{c}\text { Cogitare } \\
\text { Enfermagem }\end{array}$ & Nível 6 \\
\hline ABDEKHODA 2016 & Informática & Teerã- Irã/Hospital & $\begin{array}{l}\text { Appl Clin } \\
\text { Inform }\end{array}$ & Nível 6 \\
\hline $\begin{array}{c}\text { COSTA; PORTELA } \\
2018\end{array}$ & Odontologia & $\begin{array}{c}\text { Rio de Janeiro -RJ/ } \\
\text { Atenção Básica e } \\
\text { Hospital }\end{array}$ & $\begin{array}{l}\text { Cadernos de } \\
\text { Atenção } \\
\text { Básica }\end{array}$ & Nível 6 \\
\hline
\end{tabular}


Quadro 2 - Distribuição dos artigos segundo o título, tipo de estudo/abordagem, base de dados e objetivos dos artigos.

Perfil da equipe de enfermagem Coorte/ Definir o perfil da equipe de Enfermagem e grau de satisfação em relaçãoQuantitativa e determinar o grau de satisfação em à usabilidadedo prontuárioRelação à usabilidade do prontuário eletrônico do eletrônico do paciente paciente

A utilização do prontuárioTransversal/Caracterizar a utilização do Prontuário eletrônico do paciente porQualitativa Eletrônico do Paciente (PEP) no Hospital médicos do Hospital MunicipalMunicipal Dr. Munir Rafful, na cidade de Volta Dr. Munir Rafful: um estudo deRedonda caso

Prontuário Eletrônico: umaTransversal/Investigar a existência e a ferramenta que pode contribuiracessibilidade ao Quantitativa prontuário eletrônico para a integração das Redes de na atenção primária à saúde

Atenção à Saúde

A equipe da UTI está satisfeitaTransversal Avaliar a satisfação da equipe da com o prontuário eletrônico dounidade de terapia intensiva com o prontuário paciente? Um estudoQuantitativa eletrônico do paciente transversal

Prontuário eletrônico doTransversal/Avaliar a usabilidade e as dificuldades paciente: avaliação deQuantitativa encontradas por 99 profissionais de usabilidade pela equipe deenfermagem no manuseio de prontuário eletrônico do enfermagem. paciente.

Applying Electronic Medical Transversal/ Apresentar um modelo integrado de Records in Health Care Quantitativa aplicação de EMRs por médicos

\begin{tabular}{|c|c|c|}
\hline $\begin{array}{l}\text { Percepções de gestores, } \\
\text { profissionais e usuários acerca } \\
\text { do registro eletrônico de saúde e } \\
\text { de aspectos facilitadores e } \\
\text { barreiras para a sua } \\
\text { implementação }\end{array}$ & $\begin{array}{l}\text { Transversal/ } \\
\text { Qualitativo }\end{array}$ & $\begin{array}{l}\text { Propiciar uma visão abrangente das } \\
\text { percepções de gestores, profissionais e } \\
\text { usuários do sistema de saúde acerca de } \\
\text { suas experiências com RES }\end{array}$ \\
\hline
\end{tabular}


Quadro 3 - Contribuições do PEP para pacientes, profissionais e instituições de saúde.

CONTRIBUIÇÕES DO PEP PARA PACIENTES, PROFISSIONAIS E INSTITUIÇÕES DE SAÚDE

\section{Contribuições éticas, legais e aspectos de segurança}

- Aumento da segurança do paciente;

- Permite que as informações sejam guardadas por um período de tempo maior, garantindo maior segurança para o paciente e sua familia.

Estímulo ao trabalho em equipe, aumento da qualidade e produtividade do serviço

- Acesso remoto aos dados;

- Aumenta, significativamente, a produtividade e os índices de qualidade e satisfação dos usuários;

- Capacidade do sistema de fornecer informação clara e correta;

- Compactação do volume de dados clínicos armazenados;

- Estímulo ao trabalho colaborativo da equipe de saúde;

- Diminuição da carga de trabalho, contribuindo para qualidade do serviço.

- Eliminação da ilegibilidade da escrita a mão; 


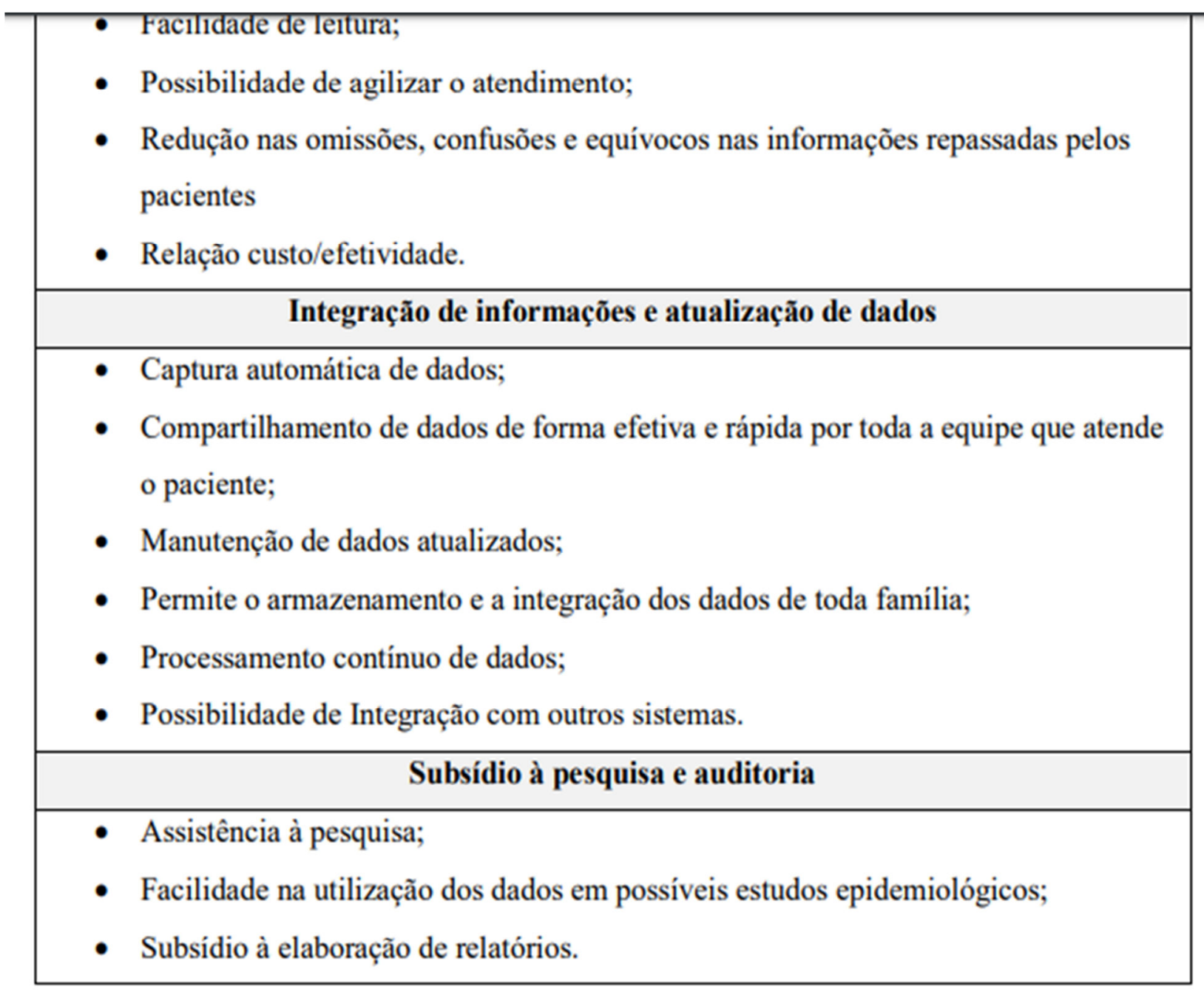

\section{DISCUSSÃO}

Os trabalhos analisados apontam contribuições importantes do PEP para pacientes, profissionais e instituições de saúde. Dentre estas, podemos citar: facilidade de leitura, aumento da segurança do paciente, acesso remoto de dados e assistência à pesquisa. O que pode ser corroborado por um estudo de revisão bibliográfica, cujo objetivo foi identificar as vantagens e desvantagens da implantação do Prontuário Eletrônico, que aponta dados semelhantes em relação às contribuições do PEP para instituições de saúde (MARTINS; LIMA, 2015).

Concernente aos níveis de evidência, constatou-se que os estudos analisados pertenciam em sua maioria ao nível 6, por tratarem-se de evidências derivadas de 
um único estudo descritivo ou qualitativo. Entretanto, não há como fazer comparações com outros estudos, visto que não foram encontrados estudos que disponibilizavam tais achados.

A produção científica referente às contribuições do PEP tem sido foco recente de atenção da equipe multidisciplinar de saúde e, principalmente dos profissionais Enfermeiros. Estes têm investido em publicações na temática, optando pela divulgação em revistas científicas indexadas em bases de dados de abrangência internacional.

Os artigos elencados mostram ainda que são vastas as contribuições obtidas com a utilização do PEP por profissionais de saúde. Nesse sentido, pode-se considerar desde a integração do PEP com outros sistemas, compactação do volume de dados armazenados, estímulo ao trabalho colaborativo em equipe, até o aumento significado da produtividade com consequente diminuição da carga de trabalho (NAMORATO et al., 2013; GONÇALVES et al., 2013).

As contribuições do PEP vão além do armazenamento de informações, exames e informações complementares, pois possibilitam armazenamento de informações por um período longo de tempo, melhoria da aderência do paciente ao tratamento, oferecendo melhores condições de atendimento a clientes em virtude da organização do serviço, com consequente impacto na qualidade de assistência ao paciente (MEDEIROS, 2014).

Desse modo, a revisão da literatura possibilitou entender que as contribuições do PEP são inúmeras, apresentando um forte argumento para que possa ser implantado nos diversos ambientes de assistência à saúde como forma de proporcionar aumento da qualidade do serviço, favorecendo assim a assistência aos usuários, o trabalho da equipe multiprofissional e o desempenho das instituições de saúde. 


\section{CONCLUSÃO}

As contribuições do PEP para pacientes, profissionais e instituições de saúde são significativas, podendo destacar contribuições éticas, legais, aspectos de segurança, estímulo ao trabalho em equipe, aumento da qualidade e produtividade do serviço, integração de informações, atualização de dados, subsídio à pesquisa e auditoria.

Contudo, percebe-se a necessidade de estudos longitudinais que permitam o acompanhamento do uso do PEP por profissionais, usuários e instituições de saúde para conhecimento de suas contribuições em longo prazo.

Portanto, a análise dos artigos mostra-se de grande relevância, uma vez que evidencia que o a utilização do PEP proporciona contribuições importantes e essenciais tanto para continuação da assistência, como para ações institucionais de saúde. 


\section{REFERÊNCIAS BIBLIOGRÁFICAS}

GANONG, L.H. Integrative reviews of nursing research. Res Nurs Health, Chichester, v.10, n.1, p.1-11, 1987.

GONÇALVES, J.P.P. et al. Prontuário Eletrônico: uma ferramenta que pode contribuir para a integração das Redes de Atenção à Saúde. Saúde em Debate, Rio de Janeiro, v. 37, n. 96, p. 43-50, 2013.

LOURENÇÃO, L.G; FERREIRA, C.J. Implantação do prontuário eletrônico do paciente no Brasil. Enferm Brasil. São Paulo, v.15, n.1, p.44-53, 2016.

MARTINS, C.; LIMA, S.M. Vantagens e desvantagens do prontuário eletrônico para instituição de saúde. RAS. São Paulo, v.16, n.63, p. 61-66, 2015.

MEDEIROS, A.L. et al. Aplicabilidade do prontuário eletrônico na área da saúde: revisão integrativa. Rev enferm UFPE on line, Recife, v.8, n.11, p. 3992-4000, 2014.

MELNYK, B.M; FINEOUT-OVERHOLT, E. Making the case for evidence-based practice. In: Melnyk, BM, Fineout-Overholt, E. Evidence-based practice in nursing \& healthcare. A guide to best practice. 2nd ed. Philadelphia: Lippincot Williams \& Wilkins; 2011.

NAMORATO, L. et al. A utilização do prontuário eletrônico do paciente por médicos do Hospital Municipal Dr. Munir Rafful: um estudo de caso. J. Health Inform, São Paulo, v.5, n.2, p.38-43, 2013.

PEREIRA, F.N. et al. Prontuário eletrônico do paciente (PEP) sua aplicabilidade e contribuição como instrumento de integração segura da informação nas redes de atendimento à saúde. Rev. Ibirapuera, São Paulo, n. 14, p. 18-26, 2017. 